\title{
Serum microRNAs improving the diagnostic accuracy in lung cancer presenting with pulmonary nodules
}

\author{
Yayi He ${ }^{1,2}$, Shengxiang Ren ${ }^{1,2}$, Yan Wang ${ }^{3}$, Xuefei Li $^{3}$, Caicun Zhou ${ }^{1}$, Fred R. Hirsch ${ }^{2}$ \\ ${ }^{1}$ Department of Medical Oncology, Shanghai Pulmonary Hospital, Tongji University Medical School Cancer Institute, Tongji University School of \\ Medicine, Shanghai 200433, China; ${ }^{2}$ Division of Medical Oncology, Department of Medicine, University of Colorado Anschutz Medical Campus, \\ Aurora, CO, USA; ${ }^{3}$ Department of Lung Cancer and Immunology, Shanghai Pulmonary Hospital, Tongji University Medical School Cancer \\ Institute, Tongji University School of Medicine, Shanghai 200433, China \\ Contributions: (I) Conception and design: C Zhou, FR Hirsch; (II) Administrative support: C Zhou, FR Hirsch; (III) Provision of study materials or \\ patients: All authors; (IV) Collection and assembly of data: Y He, S Ren, Y Wang; (V) Data analysis and interpretation: Y He, X Li; (VI) Manuscript \\ writing: All authors; (VII) Final approval of manuscript: All authors. \\ Correspondence to: Caicun Zhou. Department of Medical Oncology, Shanghai Pulmonary Hospital, Tongji University Medical School Cancer \\ Institute, Tongji University School of Medicine, Shanghai 200433, China. Email: caicunzhoudr@163.com.
}

\begin{abstract}
Background: MicroRNA (miRNA) is an approach for early diagnosing of cancer. We validated a panel of miRNAs (hsa-miR-199a-3p, hsa-miR-148a-3p, hsa-miR-210-3p, hsa-miR-378d and hsa-miR-138-5p) to aid early diagnosis of lung adenocarcinoma by blood test in lung cancer presenting with pulmonary nodules.

Methods: A total of 369 individuals who were detected pulmonary nodules by computed tomography (CT) scan were enrolled into this study. These patients included 274 pulmonary malignant or borderline lung diseases and 122 lung benign pulmonary nodules. When the lung nodules were detected by combining with CT scan, we got patient blood samples in 2 days. Patients' serum was collected within 2 days prior to miRNAs analyses. We performed miRNAs panel by reverse transcription-polymerase chain reaction (RT-PCR).

Results: The sensitivity of miRNAs panel was $34.0 \%$ and the specificity of miRNAs panel was $90.2 \%$. In invasive adenocarcinoma, the sensitivity of miRNAs panel was $44.7 \%$. The overall false positive rate of CT imaging for nodules and glass ground nodules (GGNs) was $33.1 \%$. When miRNAs panel test positive patients combined with the nodule size, the false positive rate was decreased to $3.2 \%$.

Conclusions: The greatest impact of using the miRNAs panel CT scan was decreasing the false positive. miRNAs panel can improve the diagnosis of lung cancer presenting with nodules combined with CT.
\end{abstract}

Keywords: MicroRNAs panel (miRNAs panel); diagnosis; lung cancer; nodules

Submitted Feb 05, 2018. Accepted for publication Jul 10, 2018.

doi: $10.21037 /$ jtd.2018.07.138

View this article at: http://dx.doi.org/10.21037/jtd.2018.07.138

\section{Introduction}

Lung cancer is the commonest cancer in the world (1). About 600,000 patients in China are newly diagnosed with lung cancer each year (2). Early diagnosis of lung cancer can significantly prolong survival time $(3,4)$.

Low-dose computed tomography (LDCT) has been recommended as a routine screening for lung cancer high risk patients (4). With the using of LDCT, the detection rate of lung nodules has increased year by year in China (5).
Radiology doctors preliminary differentiate benign and malignant by the density, size and shape, etc. of the pulmonary nodules (6). LDCT has a high false positive rate $(5,7)$. Some benign disease, such as sarcoidosis, atypical pneumonia, pulmonary tuberculosis, etc. could be diagnosed as malignant by radiology doctors (8). The accuracy rate of LDCT is also related to the level of doctors. Different doctors may make different judgments on the same pulmonary nodule. It is important to find a marker that can improve the diagnostic accuracy of CT. It was reported (9). 
It was reported that serum microRNAs (miRNAs) $(10,11)$, autoantibodies, circulating tumor cells $(12,13)$, and DNA methylation $(14)$, and so on $(15,16)$ could assist the diagnosis of pulmonary nodules.

miRNAs could aid to early diagnosis of cancer (15). By using a panel of six-miRNA panel (miR-429, miR-205, miR200b, miR-203, miR-125b and miR-34b) in 51 lung cancer patients, the six-miRNA panel could serve as a supplement tool for CT screening (17). Nadal et al. used a panel of four miRNAs (miR-193b, miR-301, miR-141 and miR-200b) and found four-miRNA panel discriminating lung cancer from the normal lung disease (18). These results suggested miRNAs panel test might be able to detect lung cancer. In our previously study, we developed a miRNAs panel that could aid the diagnosis of lung adenocarcinoma presenting with glass ground nodules (GGNs) by next-generation sequencing (NGS) (11). But in our previous study, the sample size was small. In this study, we expanded our sample size and used the miRNAs panel to aid CT scan. We aimed to evaluate the diagnosis accuracy of LDCT imaging combining with the miRNA panel in lung nodules.

\section{Methods}

\section{Sample collection}

From October 2015 to February 2017, 369 hospitalized patients presenting pulmonary nodules were enrolled into this study from Shanghai Pulmonary Hospital, Tongji University. Patient samples were collected in 3.8\% sodium citrate tubes. When the lung nodules were detected by CT scan, we got patient blood samples in 2 days. Most serum samples were collected within 2 days prior to the test. Samples were stored at $-80^{\circ} \mathrm{C}$. Serum miRNAs were detected by reverse transcription-polymerase chain reaction (RTPCR). Blood were collected before diagnosis and treatment. All participants provided written informed consent. All patients were diagnosed by pathology. The protocol was approved by the Shanghai Pulmonary Hospital, Tongji University (issue number 13-786, ethical number 13-768).

\section{Detected miRNA panel by qRT-PCR}

We used RT-PCR to detect the miRNA panel (hsa-miR199a-3p, hsa-miR-148a-3p, hsa-miR-210-3p, hsa-miR-378d and hsa-miR-138-5p). Relative Ct values were normalized using the U6 Ct value. Data were analyzed with the $2^{-\Delta \Delta \mathrm{Ct}}$ formula, where $\Delta \mathrm{Ct}=(\mathrm{CT}$ miRNA $-\mathrm{CTU} 6)$. Each reaction was performed in triplicate. The method was mentioned in our published paper (11). The miRNA cutoff chose the miRNA $2^{-\Delta \Delta C t}$ formula value best predicted the accuracy.

\section{Data analysis and statistics}

We did the analyses by SPSS (version 17.0, Chicago, IL, USA). We used $\chi^{2}$ tests to analyze the correlations of accuracy in different clinical parameters. $P$ values $<0.05$ (two sides) were significant.

\section{Results}

\section{Clinical data}

We collected nodule diameter, pattern, and pathology information. A total of 122 were benign pulmonary diseases; 247 were malignant or borderline diseases. Benign pulmonary diseases included pulmonary cyst, tuberculosis, granulomatous disease, pneumonia, and bronchiectasis, etc. Table 1 was the clinical data enrolled patients. In benign pulmonary diseases group, $52.5 \%$ were males and $47.5 \%$ were females; $67.2 \%$ patients were smoked; $23.0 \%$ patient GGN numbers were more than 3 . In pulmonary malignant or borderline disease group, 57.1\% were males; $57.9 \%$ were smoked; $26.3 \%$ patient GGN numbers were more than 3 .

\section{Specificities of miRNAs panel test in benign lung diseases}

The total specificity of miRNAs panel was $90.2 \%$ in benign lung diseases.

\section{Sensitivities of miRNAs panel test in lung cancer or borderline diseases}

A total of 46 patients were atypical adenomatous hyperplasia, 107 were adenocarcinoma in situ, and 85 were adenocarcinoma. The sensitivity was $44.7 \%$ in invasive adenocarcinoma (Table 2).

We did sub-analysis in patients with lung cancer, the miRNAs results were non-significant difference between sex, age, smoke status, GGNs or nodules type, and number of pulmonary nodules. The miRNAs panel positive rate was significant higher in lung GGNs or nodules patients $>8 \mathrm{~mm}$ than in patients $\leq 8 \mathrm{~mm}(\mathrm{P}=0.007)$. The positive rate of miRNAs panel in nodules group was not significantly higher than that in GGN group ( $\mathrm{P}=0.128)$ (Table 3).

We separated GGNs or nodules patients to four 
Table 1 Clinical data

\begin{tabular}{|c|c|c|c|}
\hline Characteristic & Benign pulmonary diseases, n (\%) & Malignant or borderline diseases, $\mathrm{n}(\%)$ & $\mathrm{P}$ \\
\hline Male & $64(52.5)$ & $141(57.1)$ & \\
\hline Female & $58(47.5)$ & $106(42.9)$ & \\
\hline Age (years) & & & 1.000 \\
\hline$>70$ & $13(10.7)$ & $26(10.5)$ & \\
\hline Smoker & & & 0.090 \\
\hline Smoker & $82(67.2)$ & $143(57.9)$ & \\
\hline Non-smoker & $40(32.8)$ & $104(42.1)$ & \\
\hline$>8$ & $72(59.0)$ & $134(54.3)$ & \\
\hline Number of lung GGNs or nodules, n (\%) & & & 0.526 \\
\hline$\leq 3$ & $94(77.0)$ & $182(73.7)$ & \\
\hline$>3$ & $28(23.0)$ & $65(26.3)$ & \\
\hline GGNs or nodules & & & 0.566 \\
\hline GGNs & $42(34.4)$ & $94(38.1)$ & \\
\hline Nodules & $80(65.6)$ & $153(61.9)$ & \\
\hline miRNA outcome & & & $<0.001$ \\
\hline
\end{tabular}

GGN, glass ground nodule.

groups according to their size ( $\leq 4 \mathrm{~mm}, 4<$ size $\leq 8 \mathrm{~mm},<8$ size $\leq 20 \mathrm{~mm},>20 \mathrm{~mm}$ ) and miRNAs panel in $\leq 4 \mathrm{~mm}$ group had the lowest positive rate (Figure 1, Table S1).

\section{Accuracy of miRNA panel test combined with CT imaging}

The overall false positive rate of CT imaging for nodules and GGNs was $33.1 \%$. When miRNAs panel test positive patients combined with CT imaging diagnosis in patients with various sizes of pulmonary nodules, the false positive rate was decreased to $3.2 \%$.

\section{Discussion}

Early diagnosis of lung cancer could reduce the mortality of lung cancer. National Lung Screening Trial (NLST) in the USA reported LDCT screening could reduce $20 \%$ mortality of lung cancer. Lung cancer patients diagnosed at earlier stages had a higher 5 -year survival rate (19).

More patients did the CT screening, more pulmonary nodules were found (19). CT could even detect small nodules less than one centimeter. In a large-scale study, it was pointed out that the possibility of malignancy was positively correlated with the size of the nodules (7). The Mayo Clinic CT Screening Trial reported that the nodules less than $8 \mathrm{~mm}$ had a lower malignant rate (20). The same results were also reported by Henschke et al. (21). Although the malignancy rate of small nodules is lower, there are still many small nodules are malignant, while large nodules are benign. Pathology is the gold standard for the diagnosis of pulmonary nodules. Needle biopsy in lung small nodules is a challenge for doctors, and the positive rate is relatively low. Given a 
Table 2 Sensitivities in non-small cell lung cancer

\begin{tabular}{lccc}
\hline Characteristic & miRNAs positive & Total patients & Sensitivity (\%) \\
\hline Atypical adenomatoid hyperplasia & 7 & 46 & 15.2 \\
Adenocarcinoma in situ & 37 & 107 & 34.5 \\
Invasive adenocarcinoma & 38 & 85 & 44.7 \\
Others & 2 & 9 & 22.2 \\
Total patients & 84 & 247 & 34.0 \\
\hline
\end{tabular}

Table 3 Sub-analysis in malignant or borderline diseases

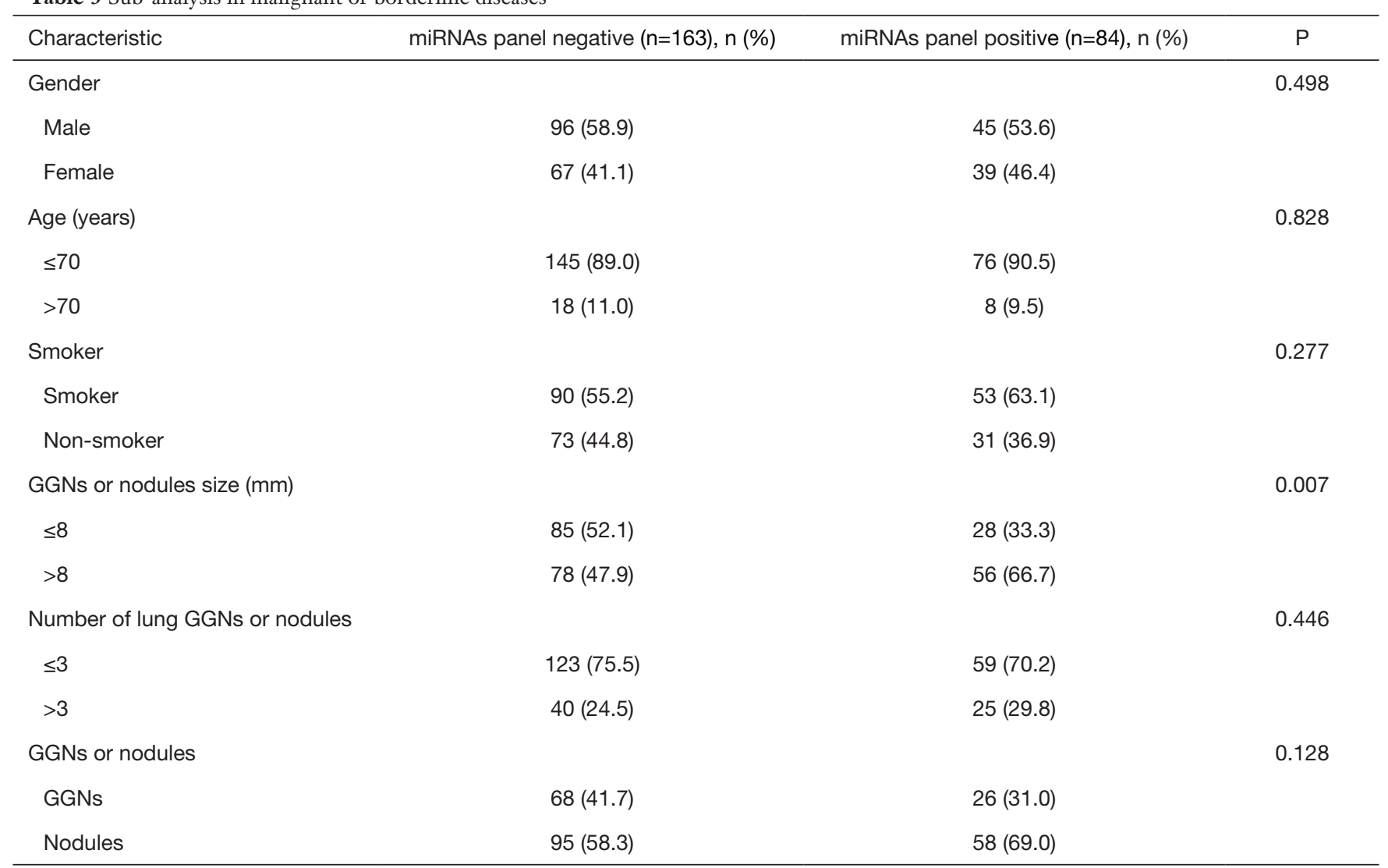

GGN, glass ground nodule.

great amount of benign nodules detected by CT scan, it is very important to find a non-invasive test which can aid CT scan to distinguish malignant diseases from benign diseases.

Liquid biopsy can assist in the diagnosis of cancer $(10,22,23)$. Cancer-associated miRNAs, autoantibodies, circulating tumor cells, and DNA methylation could be detected in patient serum, urine and plasma $(10,22,23)$. In our previously study, we found a miRNAs panel that could predict lung adenocarcinoma in GGNs patients (11). This study is to discuss the value of miRNA in assisting CT in the diagnosis of pulmonary nodules and provided the data to prove the value of using miRNA panel as biomarkers to distinguish benign nodules from malignant nodules when CT scan can only provide a rough morphology.

miRNA can be used for early diagnosis of lung cancer. In this study, we found combining miRNAs panel with LDCT scan could improve the specificity of LDCT in pulmonary nodules patients. Here we reported a miRNAs panel in patients with pulmonary nodules. The sizes between malignant and benign nodules were not 


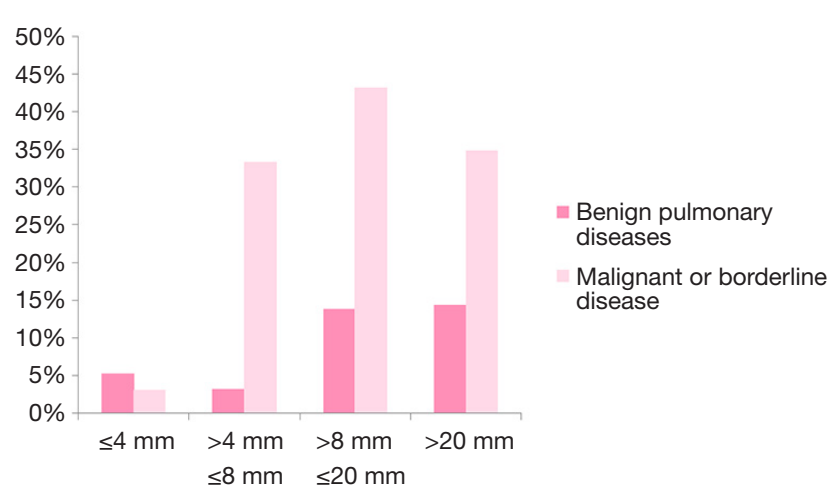

Figure 1 miRNAs panel in different nodules sizes.

significantly different. It was unreliable to judge the feature of nodules based on CT alone. Additional detection method to aid CT was needed for nodule diagnosis. In our study, 369 patient blood samples were tested by miRNAs panel. Specificity of miRNAs test was $90.2 \%$ for benign pulmonary diseases. Sensitivities of miRNAs test were $15.2 \%, 34.5 \%, 44.7 \%$, and $22.2 \%$ in atypical adenomatous hyperplasia, adenocarcinoma in situ, adenocarcinoma, and other pathologies of lung cancer. All the patients were the hospital patients. More than 30\% pulmonary nodules patients were diagnosed as the benign diseases. This study found the miRNAs panel could reduce the false positive rate of CT scan. Theoretically, miRNAs are released to the blood during growth of adenocarcinoma in situ and infiltration of cancer cells, this study suggested miRNAs panel test was a promising method for lung cancer patients with small- to medium-size pulmonary nodules. It provides a novel approach for distinguishing lung cancer from benign pulmonary diseases when CT imaging result is equivocal.

\section{Acknowledgements}

Funding: This study was supported in part by a grant from Shanghai Pujiang Program (17PJD036). Major disease clinical skills enhancement program of 3-year action plan for promoting clinical skills and clinical innovation in municipal hospitals, Shanghai Shen Kang Hospital Development Center Clinical Research Plan of SHDC (16CR1001A). The fundamental research funds for the central universities.

\section{Footnote}

Conflicts of Interest: The authors have no conflicts of interest to declare.

Ethical Statement: The study was approved by the Shanghai Pulmonary Hospital, Tongji University (issue number 13786, ethical number 13-768) and written informed consent was obtained from all patients.

\section{References}

1. Jemal A, Siegel R, Ward E, et al. Cancer statistics, 2008. CA Cancer J Clin 2008;58:71-96.

2. Chen W, Zheng R, Zhang S, et al. Lung cancer incidence and mortality in China, 2009. Thoracic Cancer 2013;4:102-8.

3. International Early Lung Cancer Action Program I, Henschke CI, Yankelevitz DF, et al. Survival of patients with stage I lung cancer detected on CT screening. N Engl J Med 2006;355:1763-71.

4. Moyer VA, Force USPST. Screening for lung cancer: U.S. Preventive Services Task Force recommendation statement. Ann Intern Med 2014;160:330-8.

5. Boiselle PM. Computed tomography screening for lung cancer. JAMA 2013;309:1163-70.

6. Horeweg N, van Rosmalen J, Heuvelmans MA, et al. Lung cancer probability in patients with CT-detected pulmonary nodules: a prespecified analysis of data from the NELSON trial of low-dose CT screening. Lancet Oncol 2014;15:1332-41.

7. Gierada DS, Pinsky P, Nath H, et al. Projected outcomes using different nodule sizes to define a positive CT lung cancer screening examination. J Natl Cancer Inst 2014;106.

8. Rothman SL, Jaffe CC, Simeone JF. Computerized tomography in the assessment of diseases of the thorax: a critical review. CRC Crit Rev Diagn Imaging 1978;11:57-74.

9. Zhi X, Shi $Y, Y u$ J. Standards for the diagnosis and treatment of primary lung cancer (2015 version) in China. Zhonghua Zhong Liu Za Zhi 2015;37:67-78.

10. Montani F, Marzi MJ, Dezi F, et al. miR-Test: a blood test for lung cancer early detection. J Natl Cancer Inst 2015;107:djv063.

11. He Y, Yang Y, Kuang P, et al. Seven-microRNA panel for lung adenocarcinoma early diagnosis in patients presenting with ground-glass nodules. Onco Targets Ther 2017;10:5915-26.

12. Wang J, Wang K, Xu J, et al. Prognostic significance of circulating tumor cells in non-small-cell lung cancer patients: a meta-analysis. PLoS One 2013;8:e78070. 
13. Juan O, Vidal J, Gisbert R, et al. Prognostic significance of circulating tumor cells in advanced non-small cell lung cancer patients treated with docetaxel and gemcitabine. Clin Transl Oncol 2014;16:637-43.

14. Mikeska T, Bock C, Do H, et al. DNA methylation biomarkers in cancer: progress towards clinical implementation. Expert Rev Mol Diagn 2012;12:473-87.

15. Solassol J, Maudelonde T, Mange A, et al. Clinical relevance of autoantibody detection in lung cancer. J Thorac Oncol 2011;6:955-62.

16. Ren S, Zhang S, Jiang T, et al. Early detection of lung cancer by using an autoantibody panel in Chinese population. Oncoimmunology 2017;7:e1384108.

17. Chen X, Jiang Y, Huang Z, et al. miRNA-378 reverses chemoresistance to cisplatin in lung adenocarcinoma cells by targeting secreted clusterin. Sci Rep 2016;6:19455.

18. Nadal E, Truini A, Nakata A, et al. A Novel Serum 4-microRNA Signature for Lung Cancer Detection. Sci Rep 2015;5:12464.

Cite this article as: He Y, Ren S, Wang Y, Li X, Zhou C, Hirsch FR. Serum microRNAs improving the diagnostic accuracy in lung cancer presenting with pulmonary nodules. J Thorac Dis 2018;10(8):5080-5085. doi: 10.21037/ jtd.2018.07.138
19. Mortani Barbosa EJ Jr. Lung cancer screening overdiagnosis: reports of overdiagnosis in screening for lung cancer are grossly exaggerated. Acad Radiol 2015;22:976-82.

20. Swensen SJ, Jett JR, Sloan JA, et al. Screening for lung cancer with low-dose spiral computed tomography. Am J Respir Crit Care Med 2002;165:508-13.

21. Henschke CI, Yankelevitz DF, Naidich DP, et al. CT screening for lung cancer: suspiciousness of nodules according to size on baseline scans. Radiology 2004;231:164-8.

22. Jett JR, Peek LJ, Fredericks L, et al. Audit of the autoantibody test, EarlyCDT(R)-lung, in 1600 patients: an evaluation of its performance in routine clinical practice. Lung Cancer 2014;83:51-5.

23. Peng J, Xie Z, Cheng L, et al. Paired design study by real-time PCR: miR-378* and miR-145 are potent early diagnostic biomarkers of human colorectal cancer. BMC Cancer 2015;15:158. 
Supplementary

Table S1 miRNAs sensitivities of CT GGNs or nodules

\begin{tabular}{lccc}
\hline GGNs or nodules size $(\mathrm{mm})$, mean & miRNAs positive & Malignant or borderline diseases & miRNAs sensitivities (\%) \\
\hline$\leq 4$ & 1 & 32 & 3.1 \\
$4<$ size $\leq 8$ & 27 & 81 & 33.3 \\
$<8$ size $\leq 20$ & 48 & 111 & 43.2 \\
$>20$ & 8 & 23 & 34.8 \\
Total & 84 & 247 & 34.0 \\
\hline
\end{tabular}

GGN, glass ground nodule; CT, computed tomography. 